\title{
Effect of Strict Lockdown on Pediatric Surgical Services and Residency Programme during COVID-19 Pandemic
}

\author{
Naima Zamir, Syed Muhammad Raees Hussain Taqvi, Jamshed Akhtar, Nasir Saleem Saddal and Muhammad \\ Anwar
}

Department of Pediatric Surgery, National Institute of Child Health, Jinnah Sindh Medical University, Karachi, Pakistan

\begin{abstract}
Objective: To document the measures adopted during the COVID-19 pandemic strict lockdown on pediatric surgical services and residents' training at a tertiary care hospital.

Study Design: Cross-sectional descriptive study.

Place and Duration of Study: Department of Pediatric Surgery, National Institute of Child Health, Jinnah Sindh Medical University, Karachi, from April 2020 to June 2020.

Methodology: Data from the Outpatient Department, Emergency Department, and Operation Theatre records were collected. The number of patients seen in the outpatient department, surgeries performed, index emergency cases dealt with, and the residents' duty roster and teaching methodology were documented. Descriptive statistics were used for reporting.

Results: During the strict lockdown period, outpatient services continued; however, elective cases were not operated. A total of 2,930 patients were seen in clinics, and 1,316 surgical procedures were performed. The index cases managed included anorectal malformation $(n=35)$, esophageal atresia with and without tracheoesophageal fistula $(n=13)$, small bowel atresia $(n=11)$, omphalocele $(n=6)$, acute appendicitis $(n=35)$, intestinal obstructions $(n=23)$, intussusceptions $(n=18)$, and intestinal perforations $(n=16)$. On-call days of residents were reduced from every third to the fifth day, and online educational sessions were added. During the pandemic, 13 members of surgical and anaesthesia teams got infected with COVID-19.

Conclusion: COVID-19 pandemic elective surgical services were restricted; however, emergency cases were managed as per routine. In clinical teaching, virtual technologies were incorporated. Working hours of residents were limited to decrease the exposure to infected persons.
\end{abstract}

Key Words: COVID-19, SARS-CoV-2 pandemic, Healthcare workers, Residency programme.

How to cite this article: Zamir N, Taqvi SMRH, Akhtar J, Saddal NS, Anwar M. Effect of Strict Lockdown on Pediatric Surgical Services and Residency Programme during COVID-19 Pandemic. J Coll Physicians Surg Pak 2021; 31(JCPSPCR):CR75-CR78.

\section{INTRODUCTION}

The COVID-19 pandemic has posed a tremendous public health threat across the globe. ${ }^{1}$ It has affected all countries worldwide, though in early months, technologically advanced countries were affected the most; but later, it equally involved countries with limited resources and the dysfunctional healthcare system. ${ }^{2}$ Different countries have used multiple strategies to deal with this catastrophe. No single approach is considered ideal, and each country has developed its own standard operating protocols, including different types of lockdowns.

Correspondence to: Dr. Naima Zamir, Department of Pediatric Surgery, National Institute of Child Health, Jinnah Sindh Medical University, Karachi, Pakistan

E-mail: naimazamir@yahoo.com

Received: September 13, 2020; Revised: March 09, 2021;

Accepted: March 25, 2021

DOI: https://doi.org/10.29271/jcpsp.2021.JCPSPCR.CR75
Most of the world literature remained focused on the medical aspect of the condition. However, surgery-related issues, especially those affecting the pediatric population, are infrequently reported. COVID-19 infection is less common in children than adults, and many of the pediatric patients are asymptomatic. ${ }^{3}$ In a study from four children hospitals in the United States, the reported incidence of COVID-19 positive surgical patients was $0.93 \% .{ }^{4}$ Surgeons are at the greatest risk, while managing patients in the current pandemic. Many surgical associations are regularly collecting data and publish updates for the surgeons to ensure their safety. ${ }^{5}$ Psychological stress of pandemic on healthcare workers (HCWs) is also reported. ${ }^{6,7}$

In Pakistan, strict lockdown was implemented in March 2020 and continued till mid of July 2020. This study was conducted to document how pediatric surgical services were provided during this time and how the residents' educational and training activities were continued. This data, from a developing and one of the most populous countries, may help public health authorities in designing protocols for pediatric surgical patients. 
Table I: Three months' pediatric surgical services data.

\begin{tabular}{|l|c|c|c|c|}
\hline Month & OPD Attendance & Main OR Surgical Procedures & \multicolumn{2}{|c|}{ Emergency OR Surgical Procedures } \\
\hline April & & GA & $64(52.5 \%)$ & $189(22.9 \%)$ \\
\hline May & $973(33.2 \%)$ & $130(33.8 \%)$ & $11(9.0 \%)$ & $258(31.3 \%)$ \\
\hline June & $74325.4)$ & $86(22.3 \%)$ & $47(38.5 \%)$ & $378(45.8 \%)$ \\
\hline Total & $1214(41.4 \%)$ & $169(43.9 \%)$ & 122 & 825 \\
\hline *Total surgical procedures performed: 1332, GA: General anesthesia, LA: Local anesthesia. & & \\
\hline
\end{tabular}

Table II: Index surgical conditions dealt with during COVID-19 pandemic $(n=216)$.

\begin{tabular}{|l|c|c|}
\hline Surgical condition & Number (n \%) & Surgical condition \\
\hline ARM: & $31(14.3)$ & Acute Appendicitis \\
High type & $4(1.9)$ & Intestinal Obstruction \\
\hline Low type & $13(6.0)$ & Intussusception \\
\hline Ssophageal atresia with / without fistula & $11(5.1)$ & Intestinal Perforation \\
\hline Malrotation & $7(3.2)$ & 23 (10.6) \\
\hline Omphalocele & $6(2.8)$ & Ventilator Dependent -Tracheostomy \\
\hline Gastroschisis & $2(0.9)$ & Abdominal Trauma \\
\hline Infantile hypertrophic pyloric stenosis & $6(2.8)$ & Irreducible Inguinal Hernia \\
\hline Posterior urethral valves & $4(1.9)$ & Pyloric Web \\
\hline Cloacal exstrophy & $1(0.5)$ & Congenital Diaphragmatic Hernia \\
\hline Bladder exstrophy & $1(0.5)$ & Meconium Ileus \\
\hline CPAM & $1(0.5)$ & Mesenteric Cyst \\
\hline Urogenital sinus anomaly & $1(0.5)$ & Torsion Testis \\
\hline
\end{tabular}

\section{METHODOLOGY}

This was a cross-sectional descriptive study. The data were collected from the surgical services provided at the Department of Pediatric Surgery, National Institute of Child Health, Jinnah Sindh Medical University, Karachi, during strict lockdown from April 2020 to June 2020. Institutional Ethical Review Board approval was obtained. Data was collected on the number of patients seen in the outpatient department, surgeries performed in emergency, and index cases operated. Residents' duty roster and educational strategies were also noted. Number of surgical, anaesthesia team members and surgical nursing and paramedical staff affected with Covid-19 infection was also recorded. Data was entered and analysed into SPSS version 22. Descriptive statistics for categorical data were presented as frequencies and percentages.

\section{RESULTS}

During the three months of the strict lockdown of COVID-19 pandemic, 2,930 patients received in the outpatient department. The number of patients increased in the third month of lockdown. Most of the surgical procedures were done as outpatient (Table I). This included suturing of traumatic wounds, drainage of abscesses, desloughing of wounds, dressings, lymph node biopsies, POP application of fractures, etc. The details of index surgical condition managed, and related surgical procedures performed are given in Table II. During the pandemic, seven doctors of the pediatric surgical and anaesthesia team were tested positive for COVID-19. Other team members included one OR nurse, one OR techni- cian, two ward nurses, one ward technician, and one sanitary worker.

Changes were made in the duty roster of surgical residents to decrease their exposure to the hospital environment. Two surgical teams were made, each working for 15 days, with every third on-call day, followed by 15 days of wash-out period. However, it was noted that residents remained away from clinical practices for an extended period that affected their training. This schedule was changed later with all residents to be present in hospital on all working days (six days a week) from 08:00 to 15:00 and attend all educational activities. However, a restricted number of residents could scrub in OR and attend outpatient clinics. The frequency of on-call duty was also reduced from every third to every fifth day. Total duty hours of residents on average were reduced from 80 hours to 65 hours per week. Additional educational activities included meeting on Zoom, and written assignments based upon clinical scenarios on WhatsApp group, specially created for this purpose. Meet the expert sessions were arranged with faculty from other hospitals. Webinars arranged by different international organisations were made mandatory to attend.

\section{DISCUSSION}

The purpose of strict lockdown during the first wave of COVID-19 pandemic was based upon the assumption that it might reduce the infection rate. Different countries have made protocols based on their circumstances. The primary mode of spread is through droplet infection. Another mode of infection in surgical practice is through aerosol generation 
procedure performed on infected patients. ${ }^{8}$ Thus, surgeons are at significant risk of contracting the infection.

Implementing strict lockdown resulted in a ban on intra-city and inter-district transport, the patients' main facility to reach the hospitals. A sharp decrease in the number of patients was noted in surgical outdoor. Before the pandemic, it was about 3,500 per month. However, during the three months of the COVID-19 pandemic, it declined to 2,930. Parents faced many challenges in terms of restriction of attendants to enter the outpatient area, mandatory wearing of masks, and rescheduling of elective admissions and surgeries. Patient-related services, especially elective surgeries, were also affected in other parts of the world, especially in early days, when there was not enough information about the COVID-19 infection. ${ }^{9}$ These services were gradually opened up with SOPs. ${ }^{10,11}$

Preventive measures have been recommended for hospitals to minimise exposure to the infection. This included a separate bay for suspected COVID patients in ER with dedicated wards for the infected patients and treatment facilities. In a systematic review, the Board of European Society of Pediatric Endoscopic Surgeons made some recommendations that included the use of disposable instrument and cables and multiple guidelines for minimally invasive procedures. ${ }^{12}$ Same approach was followed here in Pakistan though all the advanced equipment needed was not available. Training of HCWs, about protective measures is also mandatory. Emphasis was made on using proper PPE and taking precautions, while attending and operating on suspected and infected patients. During the strict lockdown period, the RT-PCR testing facility was not present at many hospitals. Preoperative testing of patients was not possible in emergency. Thus, the emphasis remained upon locally devised methods by the hospital that included wearing coverall, with N-95 mask, face shield, and limiting the number of personnel in operation theatre. The same was strictly practiced at $\mathrm{NICH}$.

The teaching and training of residents were affected during the pandemic, as there were a limited number of patients in clinics; and elective surgeries were not performed. Thus, the hands-on experience of residents was affected. In advanced countries, different computer and telephone-based educational programmes have been introduced to cater to the needs of the surgical residents. ${ }^{13,14}$ In this hospital setup, it was not possible to develop advanced simulation-based educational technologies. The residents' exposure to critical cases managed in emergency remained satisfactory, as almost all the index cases were dealt with during the pandemic. However, minimally invasive procedures were not performed as they generate aerosol and increase the risk to the surgical and anaesthesia team.

The emotional well-being of healthcare providers was another challenge in this pandemic. Physical and emotional stress has been reported in the literature. ${ }^{15}$ Considering this, counselling sessions were offered to HCWs by the hospital psychiatrist and his team. A webinar was also conducted to create awareness about the pandemic's potential effect on mental health while working under undue pressure.

An important consideration was related to pediatric patients' needs in certain conditions, where delay in treatment could have a devastating effect on the patient's health. One such area was pediatric surgical oncology. During the lockdown, tumour surgeries were limited to the diagnostic part only. Biopsies were performed either under ultrasound guidance or where lymph nodes were needed for histopathology. Oncologists made changes in chemotherapy protocols, and surgery was deferred in these patients. It was a concern that such changes may have far-reaching consequences, as reported in literature. ${ }^{16}$

In a study from South Africa, a survey was conducted on changes in surgical practices of 85 hospitals, during the hard lockdown; almost in $70 \%$ of hospitals, surgeons decided to de-escalate surgical care. ${ }^{17}$ This decision is similar to the condition here; however, the autrhors'share the same concern that the practice, on the one hand, affected the care of surgical patients as they were not followed regularly; and on the other hand, it has created a backlog of surgical procedures to be performed once surgical services shall be resumed. Thus, the challenge will be huge for countries with limited surgical facilities.

Overall, several strategies were made to balance the needs of the patient population, residents in training, and the most essential, the safety of healthcare providers who were the front-line soldiers against a covert enemy. No single approach can be recommended to take care of all the needs. A flexible programme with frequent changes based upon ground realities, available resources, and evolving knowledge may be the most appropriate way forward. Documentation of such experiences also helps others to devise strategies.

\section{CONCLUSION}

The number of patients attending the outpatient department was limited, but emergency cases remained adequate with a wide range of anomalies managed during strict lockdown period. In clinical teaching, virtual technologies were incorporated along with frequent changes in residents' duties as the pandemic progressed. The emphasis remained on observing SOPs and using all personal protective measures to combat the challenges.

\section{ETHICAL APPROVAL:}

Institutional Ethical Review Board approval was obtained from the Department of Pediatric Surgery, National Institute of Child Health, Jinnah Sindh Medical University, Karachi.

\section{PATIENTS' CONSENT:}


The IERB granted a waiver for the informed consent as the study did not involve any interaction with human participants.

\section{CONFLICT OF INTEREST:}

The authors declared no conflict of interest.

\section{AUTHORS' CONTRIBUTION:}

NZ: Concept, critical review of manuscript and final approval. SMRHT: Critical review of manuscript and final approval. JA: Conception, literature search, data collection, manuscript writing, and final approval of draft.

NSS, MA: Critical review of manuscript and final approval.

\section{REFERENCES}

1. World Health Organization. Coronavirus disease (COVID-19), Situation Report-159. http://www.who. int/docs/default-source/coronaviruse/situation-reports/20200627-covid-19-sitrep-159.pdf?sfvrsn=93e027f6 2. Published June 27, 2020. (accessed on June 30, 2020).

2. Abid K, Bari YA, Younas M, Tahir Javaid S, Imran A. Progress of COVID-19 Epidemic in Pakistan. Asia Pac J Public Health. 2020; 32(4):154-6. doi: 10.1177/101053 9520927259.

3. Zhou Y, Xu H, Li Long, Ren X. Management for patients with pediatric surgical disease during the COVID-19 epidemic. Pediatr Surg Int 2020; 36:751-2. doi: 10.1007/ s00383-020- 04656-6.

4. Lin EE, Blumberg TJ, Adler AC, Fazal FZ, Talwar D, Ellingsen $\mathrm{K}$, et al. Incidence of COVID-19 in pediatric surgical patients among 3 US children's hospitals. JAMA Surg 2020; doi:10.1001/jamasurg.2020.2588.

5. American College of Surgeons. COVID-19: Considerations for optimum surgeon protection before, during, and after operation. Updated on April 1, 2020. http://www.facs.org/ covid-19/clinical-guidance/surgeon-protection (accessed on June 30, 2020).

6. Osama M, Zaheer F, Saeed H, Anees K, Jawed Q, Syed SH, et al. Impact of COVID-19 on surgical residency programs in Pakistan; A residents' perspective. Do programs need formal restructuring to adjust with the "new normal"? A cross-sectional survey study. Int J Surg 2020; 79:252-6. doi: 10.1016/j.ijsu.2020.06.004.

7. He K, Stolarski A, Whang E, Kristo G. Addressing general surgery residents' concerns in the early phase of the COVID-19 pandemic. J Surg Educ. 2020; 77:735-8. doi:10. 1016/ j.jsurg.2020.04.003.

8. Francom CR, Javia LR, Wolter NE, Lee GS, Wine T,
Morrissey $\mathrm{T}$, et al. Pediatric laryngoscopy and bronchoscopy during the COVID-19 pandemic: A four-center collaborative protocol to improve safety with perioperative management strategies and creation of a surgical tent with disposable drapes. Int J Pediatr Otorhinolaryngol 2020; 134:110059. doi:10.1016/j.ijporl.2020.110059.

9. Nair AG, Gandhi RA, Natarajan S. Effect of COVID-19 Related lockdown on ophthalmic practice and patient care in India: Results of a survey. Indian J Ophthalmol. 2020; 68:725-730. doi: 10.4103/ijo.IJO_797_20.

10. Royal College of Surgeons England. Recovery of surgical services during and after COVID-19. http://www.rcseng. ac.uk/coronavirus/recovery-of-surgical-services/ (accessed on July 1, 2020).

11. Coimbra R, Edwards S, Coimbra BC, Bass GA, Balogh ZJ, Tilsed J, et al. Resuming elective surgical services in times of COVID-19 infection. Trauma Surg Acute Care Open 2020; 5(1):e000511. doi: 10.1136/tsaco-2020-000511.

12. Pini Prato A, Conforti A, Almstrom M, Gemert WV, Scuderi MG, Khen-Dunlop N, et al. Management of COVID-19-positive pediatric patients undergoing minimally invasive surgical procedures: systematic review and recommendations of the Board of European Society of Pediatric Endoscopic Surgeons. Front Pediatr 2020; 8:259. doi:10.3389/fped. 2020.00259.

13. McKenchnie T, Levin M, Zhou K, Freedman B, Palter V, Grantcharov TP. Virtual surgical training during COVID-19. Operating room simulation platforms accessible from home. Ann Surg 2020; 272(2):e153-4. doi: 10.1097/SLA. 0000000000003999.

14. Ehrlich $\mathrm{H}$, McKenney M, Elkbuli A. We asked the experts: Virtual learning in surgical education during the covid-19 pandemic-shaping the future of surgical education and training. World J Surg 2020; 44(7):2053-5. doi:10.1007/ s00268-020-05574-3.

15. Murphy B. Residency in a pandemic: How COVID-19 is affecting trainees. American Medical Association. ttps:// www.ama-assn.org/residents-students/residency/residenc y-pandemic-how-covid-19-affecting-trainees (accessed on July 2, 2020).

16. Søreide K, Hallet J, Matthews JB, Schnitzbauer AA, Line PD, Lai PBS, et al. Immediate and long-term impact of the COVID-19 pandemic on delivery of surgical services. $\mathrm{Br} J$ Surg 2020; 10.1002/bjs.11670. doi:10.1002/bjs.11670.

17. Chu KM, Steyn E, Goldberg P, Goldberg P, Bougard H, Buccimazzaet I. Changes in surgical practice in 84 South African hospitals during Covid-19 hard lockdown. South Afr Med J. 2020; doi.org/10.7196/SAMJ.2020.v110i9.15014. 